Novartis, Ono, Pfizer, Sanofi and Takeda, Grant/research support from: Asahi Kasei, Abbvie, Chugai, Eisai, Yves Schymura Employee of: Eli Lilly, Andrew Bradley Shareholder of: Eli Lilly, Employee of: Eli Lilly, Jens Gerwien Shareholder of: Eli Lilly, Employee of: Eli Lilly, Brigitte Monsberger Shareholder of: Eli Lilly, Employee of: Eli Lilly, Soyi Liu Leage Employee of: Eli Lilly, Daniel Aletaha Speakers bureau: Abbvie, Amgen, Celgene, Lilly, Medac, Merck, Novartis, Pfizer, Roche, Sandoz, Sanofi/Genzyme, Grant/research support from: Abbvie, Lilly, Novartis, Roche, Mikkel Østergaard Speakers bureau: Abbvie, Celgene, Eli-Lilly, Janssen, Novartis, Pfizer, Roche, Sanofi and UCB, Consultant of: Abbvie, BMS, Boehringer-Ingelheim, Celgene, Eli-Lilly, Hospira, Janssen, Merck, Novartis, Novo, Orion, Pfizer, Regeneron, Roche, Sandoz, Sanofi and UCB, Grant/research support from: Abbvie, BMS, Merck, Celgene, Novartis DOI: 10.1136/annrheumdis-2021-eular.2079

\begin{tabular}{|l|l|}
\hline POS0927 & EFFECTIVENESS AND SAFETY OF SECUKINUMAB \\
& IN NAIIVE OR TNF-INHIBITORS FAILURE AXIAL \\
& SPONDYLOARTHRITIS PATIENTS IN REAL LIFE: A \\
& 24-MONTHS PROSPECTIVE MULTICENTRIC STUDY
\end{tabular}

M. Lorenzin ${ }^{1}$, A. Ortolan ${ }^{1}$, M. S. Chimenti ${ }^{2}$, A. Marchesoni ${ }^{3}$, E. Lubrano ${ }^{4}$ L. Santo ${ }^{5}$, A. Semeraro ${ }^{6}$, C. Salvarani ${ }^{7,8}$, N. Girolimetto ${ }^{8}$, E. Praino ${ }^{9}$, G. L. Fonti ${ }^{2}$, R. Foti ${ }^{10}$, A. Carletto ${ }^{11}$, A. Doria ${ }^{12}$, R. Ramonda ${ }^{12}$ On behalf of Italian Society of Rheumatology (SIR) "Spondyloartritis and Psoriatic Arthritis Study Group-A. Spadaro.' ' University of Padova, Rheumatology Unit, Department of Medicine DIMED, Padova, Italy; ${ }^{2}$ University of Rome "Tor Vergata", Rheumatology, Allergology and Clinical Immunology Department of "Medicina dei Sistemi", Roma, Italy; ${ }^{3}$ ASST Gaetano Pini-CTO, Milan, Department of Rheumatology, Milano, Italy; ${ }^{4}$ University of Molise, Campobasso, Academic Rheumatology Unit, Dipartimento di Medicina e Scienze per la Salute "Vincenzo Tiberio", Campobasso, Italy; ${ }^{5}$ ASL BT Andria - DSS4 Barletta, Italy, Barletta-Andria-Trani, Rheumatology Unit, Barletta-Andria-Trani, Italy; ${ }^{6}$ Martina Franca-ASL Taranto, Rheumatology Unit, Taranto, Italy; ${ }^{7}$ University of Modena and Reggio Emilia, Rheumatology Unit, Reggio Emilia, Italy; ${ }^{8}$ Azienda USL-IRCCS, Istituto di Ricovero e Cura a Carattere Scientifico, Rheumatology Unit, Department of Internal Medicine, Reggio Emilia, Italy; ${ }^{9}$ ASL BT Andria - DSS4 Barletta, Italy, Barletta-Andria-Trani, Rheumatology Unit, Barletta-Andria-Trani, Italy; ${ }^{10}$ A. O.U. Policlinico S. Marco, Catania, Italy, Rheumatology Unit, Catania, Italy; ${ }^{11}$ AOUI University of Verona, Rheumatology Unit, Department of Medicine, Verona, Italy; ${ }^{1}$ University of Padova, Rheumatology Unit, Department of Medicine DIMED, Padova, Italy

Background: Axial Spondyloarthritis (axSpA) can be distinguished in radiographic axSpA (r-axSpA) and non-radiographic (nr-axSpA). Secukinumab (SEC) is a novel treatment for axSpA, but data from real-life are still missing.

Objectives: 1)to evaluate the effectiveness and safety of a wide cohort of axSpA patients on SEC followed in 8 Italian Rheumatologic centers for 24-months;2) to compare the features and disease-activity indices of SEC-treated axSpA patients subdivided in naïve biological drugs (group A) and in TNF-inhibitors failure patients (group B).

Methods: Consecutive patients with active axSpA (diagnosis according Assessment of SpondyloArthritis International Society ASAS criteria), who started SEC treatment, were evaluated prospectively.Data on disease characteristics, previous/ongoing treatments and imaging were collected. Disease-activity/functional/ clinical scores and biochemical values were recorded at baseline (T0), at 6 (T6), 12 (T12), and 24 (T24) months. Effectiveness was evaluated over-time with descriptive statistics. Anova (Kruskal Wallis) and generalized linear models were used to compare variables over-time. Infections, adverse events were collected. Results: One-hundred-seven patients [49.53\% men; median age 49years; median treatment duration 18.5years] were enrolled;53(49.53\%) had HLA-B27, 47.66\% were $\mathrm{r}$-axSpA and $52.34 \% \mathrm{nr}$-axSpA. Signs of sacroiliitis were present on MRI in $97(90.65 \%)$ and X-rays in $51(47.66 \%)$. SEC was prescribed as first line biologic treatment in $32(29.9 \%)$ patients and as second or more line biological treatment in $75(70.1 \%)$ patients (Figure 1). In all population significant decrease was achieved in:Visual Analogue Scale of pain and general-health; Leeds Enthesitis Index; Health Assessment Questionnaire modified for spondyloarthritis (HAQ-s);Bath Ankylosing Spondylitis Functional Index (BASFI);C-reactive protein. Bath Ankylosing Spondylitis Metrology Index and Erythrocyte-sedimentation-rate not significantly decreased. Effectiveness was associated to an improvement in Ankylosing Spondylitis disease activity score (ASDAS) [T0=3.4 (2.9-3.9) vs T24=1.9 (1.2-2.7); $\mathrm{p}=0.02]$ and in Bath Ankylosing Spondylitis Disease Activity Index (BASDAI) $[$ T0=6.6 (5.0-7.8) vs T24=3.2 (2.0-5.0); $p=0.03$ ].At T0 group $B$ had a longer disease duration $(p=0.04)$, a greater prevalence of peripheral arthritis $(p=0.02)$,enthesitis $(p=0.04)$ and psoriasis $(p=0.05)$ and was mostly male $(p=0.05)$, while no significant difference was observed for functional and disease-activity indices and signs of sacroilitis on MRI/X-rays. At T24 group A showed better physical functioning and lower disease activity compared to group $B$ [HAQs A vs. $B=0.1(0.0-0.5)$ vs $0.3(0.1-0.8)$; $B A S F I A$ vs $B=1.6(0.8-4.8)$ vs 4.0(2.5-4.6); BASDAI A vs $B=2.2(1.0-3.8)$ vs 3.9(2.7-5.0);ASDAS A vs $B=1.3(1.0-2.2)$ vs 2.1(1.6-2.9)].After T24 of treatment $70.2 \%$ of Group A and $68.4 \%$ of Group B had a low disease activity,accordingly to ASDAS<2.1. Twenty-three patients $(21.5 \%)$ stopped the treatment during the follow-up mainly because of primary (7) or secondary loss of efficacy (9). Only 7 patients suspended SEC because of adverse events.A low number of episodes of mild infections (19) occurred;SEC was instead permanently discontinued in 4 cases for:oral refractory mucositis (2);recurrent aphthosis (1);recurren broncopneumoniae (1).The retention rate at 24 was good in the whole population (73\%).Survival curves for Group A and B were similar (log-rank test=0.81; $=0.69$ ). Conclusion: In a real-life clinical setting,SEC was safe and effective in axSpA, as shown by a significant decrease of BASDAI and ASDAS over a 24-months follow-up.

Baseline characteristics of 107 axSpA patients treated with secukinumab in the period January 2017- December 2020

\begin{tabular}{|c|c|c|c|c|}
\hline axSpA Features & Total patients & GROUP A vs. & GROUP B & p \\
\hline Male sex $(\mathrm{N}, \mathrm{S})$ & $53(49.53 \%)$ & $23(71.88 \%)$ & $30(40 \%)$ & 0.05 \\
\hline Age (years), median (IOR) & $49(40.5-58)$ & $44(35.75-58)$ & $52(42-58.5)$ & 0.04 \\
\hline Age of diagnosis (years), median (IOR) & $43(32.5-51)$ & $39.5(305-50)$ & $43(33.5-53)$ & ns \\
\hline Age of disease onset (years), median (IQR) & $39(29.5-49)$ & $36.5(22.75-46)$ & $40(30.5 .49)$ & ns \\
\hline Disease duration (years), median (IOR) & $9(4-13.5)$ & $7(2-14.25)$ & $10(6-13)$ & 0.04 \\
\hline $\operatorname{axSpA}(n, \%)$ & 107 & $32(29.91 \%)$ & 75 (70.09\%) & NA \\
\hline$r-a \times S p A$ & $51(47.66 \%)$ & 15 (46.88\%) & $36(48 \%)$ & ns \\
\hline$n r-a x S \rho A$ & $56(52.34 \%)$ & $17(53.13 \%)$ & $39(52 \%)$ & ns \\
\hline HLA-B27 positive, $\mathrm{N}(\%)$ & $53(49.53 \%)$ & $16(50 \%)$ & $37(49.33 \%)$ & ns \\
\hline SI-MRI positive, N (\$) & $97(90.65 \%)$ & $28(87.5 \%)$ & $69(92 \%)$ & ns \\
\hline Su-X-rays positive, $\mathrm{N}(\mathrm{S})$ & $51(47.66 \%)$ & $15(46.9 \%)$ & $36(48 \%)$ & ns \\
\hline Peripheral arthritis, $\mathrm{N}(\boldsymbol{\%})$ & 62 (57.94\%) & $15(46.9 \%)$ & $47(62.67 \%)$ & 0.02 \\
\hline Enthesitis, $\mathbf{N}(\mathbf{\%})$ & $66(61.68 \%)$ & $17(53.13 \%)$ & $49(65.33 \%)$ & 0.04 \\
\hline Dactylitis, $N(\%)$ & 14 (13.08\%) & $5(15.63 \%)$ & $9(12 \%)$ & ns \\
\hline Psoriasis, N (\%) & $50(46.73 \%)$ & $13(40.63 \%)$ & $37(49.33 \%)$ & 0.05 \\
\hline Onicopathy, $\mathrm{N}(\mathrm{\%})$ & $22(20.56 \%)$ & $6(18.75 \%)$ & $16(21.33 \%)$ & ns \\
\hline $\mathrm{IBD}, \mathrm{N}(\%)$ & $4(3.74 \%)$ & $2(6.25 \%)$ & $2(2.67 \%)$ & ns \\
\hline Uveitis, N (\$) & $5(4.67 \%)$ & $0(0 \%)$ & $5(6.67 \%)$ & ns \\
\hline Familiarity with psoriasis or SpA & 36 (33.64\%) & $12(37.5 \%)$ & $24(32 \%)$ & ns \\
\hline Smoking, $N(\%)$ & $26(24.30 \%)$ & $8(25 \%)$ & $18(24 \%)$ & ns \\
\hline Weight (kg), median (IOR) & $70(60.5-82)$ & $70.50(64.5-82.25)$ & $70(60-81.5)$ & ns \\
\hline Height (cm), median (IOR) & $169(161-177)$ & $174(165-178)$ & $168(160-175.5)$ & ns \\
\hline BMI, median (IQR) & $24.69(21.95-28.05)$ & $23.97(22.65-26.20)$ & $24.9(21.79-28.24)$ & ns \\
\hline BASMI [0-10], median (IOR) & $3(1-6)$ & $2(0-4)$ & $4(1-7)$ & 0.03 \\
\hline LE [0-6], median (IOR) & $1(0-2)$ & $0(0-1.5)$ & $1(0-2)$ & ns \\
\hline ESR [0-25] (mm/h), median (IOR) & $16(9-28)$ & $13.5(5-27.25)$ & $18.5(10-28)$ & ns \\
\hline CRP $[0-6](\mathrm{mg} / \mathrm{L})$, median (IQR) & $5(2.9 \cdot 11)$ & $3.45(2.4-15.3)$ & $5(2.9-11)$ & 0.04 \\
\hline VAS-pain [0-10], median (IOR) & $7(6-8)$ & $6.65(6-7.45)$ & $7(6-8)$ & ns \\
\hline VAS-gh [0-10], median (IOR) & $5.6(4-7)$ & $5.0(4.25-7.0)$ & $6(4-7.1)$ & ns \\
\hline HAQ-S [0-8], median (IOR) & $1(0.5-1.5)$ & $0.75(0.2-1.0)$ & $1(0.75-1.50)$ & 0.05 \\
\hline BASFI [0-10], median (IQR) & $6(4.95-7.03)$ & $5.5(3.63-7.05)$ & $6(5.1-7.0)$ & ns \\
\hline BASDAI [0-10], median (IOR) & $6.6(5-7.8)$ & $6(3.7-6.95)$ & $7(5.25-8.2)$ & ns \\
\hline ASDAS [0-6], median (IOR) & $3.35(2.85-3.86)$ & $3.33(2.67-3.68)$ & $3.35(2.90-3.86)$ & ns \\
\hline Mean treatment duration (months), median (IOR) & $18.5(12-32.5)$ & $18.10(12-30)$ & $18.93(12-32.5)$ & ns \\
\hline Dosage $300 \mathrm{mg} /$ injection $(\mathrm{N}, \mathrm{S})$ & $33(30.84 \%)$ & $6(18.75 \%)$ & $27(36 \%)$ & 0.04 \\
\hline Dosage $150 \mathrm{mg} /$ injection $(\mathrm{N}, \%)$ & 74 (69.16\%) & $26(81.25 \%)$ & $48(64 \%)$ & 0.04 \\
\hline $1^{\prime \prime}$ line $(N, \%)$ & 32 (29.91\%) & $32(100 \%)$ & $0(0 \%)$ & NA \\
\hline Failure biological drugs $(\mathrm{N}, \%)$ & 75 (70.10\%) & $0(0 \%)$ & $75(100 \%)$ & NA \\
\hline $2^{\text {nd line }}(\mathrm{N}, \%)$ & 33 (30.84\%) & $0(0 \%)$ & $33(44 \%)$ & NA \\
\hline $3^{\text {rt }}$ line $(N, \%)$ & $23(21.50 \%)$ & $0(086)$ & $23(30.67 \%)$ & NA \\
\hline $4^{\text {th }}$ line $(N, \%)$ & $13(12.15 \%)$ & 0 (0\%) & $13(17.33 \%)$ & NA \\
\hline $25^{\mathrm{th}}$ line $(\mathrm{N}, \%)$ & $6(5.61 \%)$ & $0(0 \%)$ & $6(8 \%)$ & NA \\
\hline Concomitant NSAIDS, N (\%) & $68(63.55 \%)$ & $21(65.63 \%)$ & $47(62.67 \%)$ & ns \\
\hline Concomitant glucocorticosteroid, $\mathrm{N}(\%)$ & 22 (20.56\%) & $6(18.75 \%)$ & 16 (21.33\%) & ns \\
\hline Concomitant CsDMARDs, $\mathbf{N}(\%)$ & $23(21.50 \%)$ & $6(18.75 \%)$ & $17(22.67 \%)$ & \\
\hline \multicolumn{5}{|c|}{ 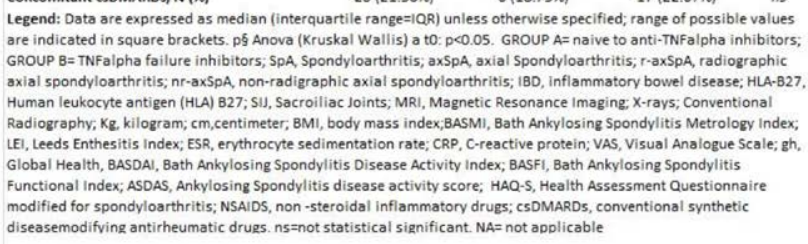 } \\
\hline
\end{tabular}

Disclosure of Interests: Mariagrazia Lorenzin: None declared, Augusta Ortolan: None declared, Maria Sole Chimenti: None declared, Antonio Marchesoni Grant/research support from: AM has received honoraria and speaker fees from Abbvie, Pfizer, MSD, UCB, Novartis, Janssen, Eli-Lilly., Ennio Lubrano: None declared, Leonardo Santo Speakers bureau: Speaker from Jansen, Novartis, Pfizer, UCB, MSD, Sanofi, Angelo Semeraro: None declared, Carlo Salvarani: None declared, Nicolò Girolimetto: None declared, Emanuela Praino: None declared, Giulia Lavinia Fonti: None declared Rosario Foti: None declared, Antonio Carletto: None declared, Andrea Doria Grant/ research support from: ADhas received honoraria and speaker fees from Novartis Abbvie, Pfizer, MSD, Janssen., Roberta Ramonda Grant/research support from: RR has received honoraria and speaker fees from Novartis, Abbvie, Pfizer, MSD, Janssen. DOI: 10.1136/annrheumdis-2021-eular.2121

\section{POS0928 NETAKIMAB EFFICACY IN ANTI-TNF-NAIVE AND ANTI-TNF-EXPERIENCED PATIENTS WITH ACTIVE ANKYLOSING SPONDYLITIS: RESULTS OF SUBANALYSIS OF PHASE 3 ASTERA TRIAL}

S. Erdes ${ }^{1}$, V. Mazurov ${ }^{2}$, T. Dubinina ${ }^{3}$, I. Gaydukova ${ }^{2,4}$, A. Kundzer ${ }^{5}$, N. Soroka ${ }^{6}$, A. Eremeeva ${ }^{7} .{ }^{1}$ Nasonova Research Institute of Rheumatology, Laboratory of Spondyloarthritides, Moscow, Russian Federation; ${ }^{2}$ Mechnikov NorthWestern State Medical University, Department of Therapy and Rheumatology 